\title{
Treatment of an Atypical Case of Bilateral Nephrolithiasis in a Canine
}

\author{
Inácio Bernhardt Rovaris', Aline Silva Gouvêa ${ }^{2}$, Tainor de Mesquita Tisotti ${ }^{3}$, \\ Gabriela da Cruz Schaefer', Eduardo Raposo Monteiro ${ }^{4}$ \& Marcelo Meller Alievi ${ }^{4}$
}

\begin{abstract}
Background: Less than 5\% of canine uroliths are found in the kidney and ureter. The decision to remove a nephrolith is controversial and should be considered in cases of refractory infection, hematuria, presence of obstructive uropathy and compression of the renal parenchyma. The aim of this report is to describe an unusual presentation of bilateral nephrolithiasis in a dog, occupying almost the entire renal parenchyma, its clinical and imaging findings, in addition to surgical treatment and its evolution.

Case: A 10-year-old male Basset Hound was evaluated at the Veterinary Medical Teaching Hospital (HCV) of the Federal University of Rio Grande do Sul (UFRGS), presenting prostration, anorexia, vomiting, diarrhea and severe hematuria for three days. On physical examination the patient was dehydrated, with pale mucous membranes, uremic breath and abdominal pain. Blood tests showed mild anemia and azotemia. In both kidneys, abdominal ultrasonography exhibited a large hyperechoic structure with deep acoustic shadowing. These same structures were observed in abdominal radiographic examination as radiopaque structures, confirming the diagnosis of bilateral nephrolithiasis. Urine culture was positive for coagulase-negative Staphylococcus sp. The patient was stabilized with fluid therapy, antiemetic, analgesics, antibiotics and whole blood transfusion. Unilateral nephrotomy of the right kidney was performed to remove the urolith. After three months, nephrotomy of the left kidney was performed to remove the other urolith. The patient was clinically stable and with no macroscopic hematuria $12 \mathrm{~h}$ after surgery. Two days after discharge, the patient returned prostrated in lateral recumbency, however with no alteration of parameters in physical examination. Blood tests showed anemia, thrombocytopenia, hypoalbuminemia, azotemia and hyperphosphatemia. The dog presented a convulsive episode and died shortly, eight hours later. In the necropsy examination, extensive loss of renal parenchyma was seen in both kidneys and the presence of thrombosis and areas of infarction in several organs such as spleen, liver, lungs and central nervous system.

Discussion: Complications of nephrotomy include perirenal hemorrhage, hydronephrosis due to obstruction of blood clots or urine leakage to the abdominal cavity. From the necropsy findings, the surgical site did not present these complications, justifying that the surgical procedure was not directly related to death. Correction of dehydration, blood transfusion, and antibiotic therapy based on urine culture and susceptibility testing was important for initial stabilization, before surgery. However, the presence of advanced chronic kidney disease may have contributed to deterioration of the patient's clinical condition and death. The presence of thrombus and hemorrhagic areas, observed during necropsy, associated with acute onset of clinical signs and the presence of severe hypoalbuminemia, may suggest that the patient died due to a thromboembolic event, as a consequence of nephrotic syndrome. Studies show that nephrotomy can be performed with few adverse effects on renal function if the surgical technique and anesthetic management are adequate. In this case, nephrotomy was the best option for the treatment of the patient, since it enabled a rapid intervention, controlled the hematuria and allowed the removal of both uroliths without complications related to surgery.
\end{abstract}

Keywords: nephrotomy, urolithiasis, kidney, surgery. 


\section{INTRODUCTION}

Less than $5 \%$ of canine uroliths are found in the kidney and ureter [7]. Nephrolithiasis may be asymptomatic or present with pain, fever, hematuria and/or signs of renal dysfunction [5]. Bilateral renal uroliths may be present in about $20 \%$ of the dogs diagnosed with nephrolithiasis [5] and struvite and calcium oxalate are the most common types in this species [7]. Factors contributing to urolith formation include urine $\mathrm{pH}$, high urine specific gravity, presence of urinary tract infection, presence of lithogenic substances in urine and decrease of inhibitors of crystallization [1]. Determining the composition of uroliths is essential to prevent recurrence [1].

The decision to remove a nephrolith is controversial and should be considered in cases of refractory infection, hematuria, presence of obstructive uropathy and compression of the renal parenchyma [4]. The aim of this report is to describe an unusual presentation of bilateral nephrolithiasis in a dog, occupying almost the entire renal parenchyma, its clinical and imaging findings, in addition to surgical treatment and its evolution.

\section{CASE}

A 10-year-old male Basset Hound was evaluated in the Veterinary Teaching Hospital of Federal University of Rio Grande do Sul, presenting prostration, anorexia, vomiting, diarrhea and severe hematuria for three days. The dog had an episode of hematuria 30 days ago and was treated with meloxicam, cephalexin and fluid therapy, with improvement after treatment. On physical examination the patient was dehydrated, with pale mucous membranes, uremic breath and abdominal pain. A complete blood count showed mild anemia (hematocrit of $25 \%$ and total plasma protein of $80 \mathrm{~g} / \mathrm{L}$ ) and the biochemical evaluation revealed azotemia (creatinine $371.3 \mu \mathrm{mol} / \mathrm{L}$ and urea $53.7 \mathrm{mmol} / \mathrm{L}$ ). In both kidneys, abdominal ultrasonography exhibited a large hyperechoic structure with deep acoustic shadowing in the topography of medullar region, in addition to reduced renal parenchyma. The right ureter was dilated $(0.53 \mathrm{~cm})$. These same structures were observed in abdominal radiographic examination (ventrodorsal and lateral views) as radiopaque structures (Figure $1 \mathrm{AB}$ ), confirming the diagnosis of bilateral nephrolithiasis. Urinalysis revealed a red and turbid urine. Urine specific gravity was 1.012 and urine $\mathrm{pH}$ was

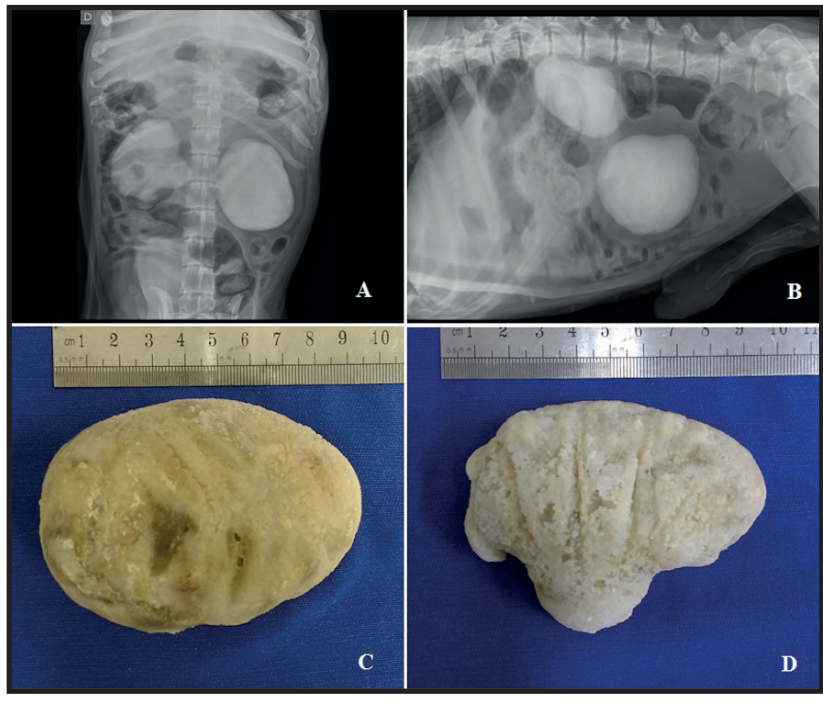

Figure 1. Abdominal radiography on ventrodorsal view (A) and lateral view (B), showing the presence of large radiopaque structures (uroliths) in both kidneys. Right nephrolith (C) and left nephrolith (D) after surgical removal.

8.0. Evaluation of urine sediment showed proteinuria $(3+)$, bacteriuria (3+), presence of squamous epithelial cells and transitional epithelial cells, in addition to countless leukocytes and erythrocytes. Urine culture was positive for coagulase-negative Staphylococcus sp., susceptible to multiple classes of antibiotics. The patient remained hospitalized receiving fluid therapy with lactated Ringer's solution ${ }^{1}$ (40 mL/kg/day IV), omeprazole $^{2}$ (1 mg/kg q 24h IV), maropitant ${ }^{3}(1 \mathrm{mg} / \mathrm{kg}$ q $24 \mathrm{~h} \mathrm{SC}$ ) and tramadol ${ }^{4}(3 \mathrm{mg} / \mathrm{kg} \mathrm{q} 8 \mathrm{~h} \mathrm{SC}$ ). Enrofloxa$\operatorname{cin}^{5}(5 \mathrm{mg} / \mathrm{kg}$ q $24 \mathrm{~h} \mathrm{IV}$ ) was prescribed based on urine culture and sensitivity testing. The dog presented a persistent azotemia (creatinine $450.8 \mu \mathrm{mol} / \mathrm{L}$ and urea $52.5 \mathrm{mmol} / \mathrm{L}$ ). Whole blood transfusion was performed for pre-surgical stabilization, due to the persistence of severe hematuria and decrease in hematocrit to $15 \%$ (without evidence of regeneration).

Unilateral nephrotomy of the right kidney was performed due to the smaller dimensions of this urolith and the greater amount of remaining parenchyma, compared to the contralateral kidney. Methadone ${ }^{6}$ $(0.2 \mathrm{mg} / \mathrm{kg} \mathrm{IM})$ was used as preanesthetic medication and the patient was pre-oxygenated. Anesthetic induction was performed with propofol ${ }^{6}(4 \mathrm{mg} / \mathrm{kg} \mathrm{IV})$ and maintenance with isoflurane ${ }^{6}$. The patient was placed in dorsal recumbency and a ventral midline incision from the xiphoid process of the sternum to five centimeters caudal to the umbilicus was performed. Markedly enlarged and firm kidneys were noted (Figure 2A). The peritoneum surrounding the right kidney was carefully dissected, facilitating the exposure and isolation of the 


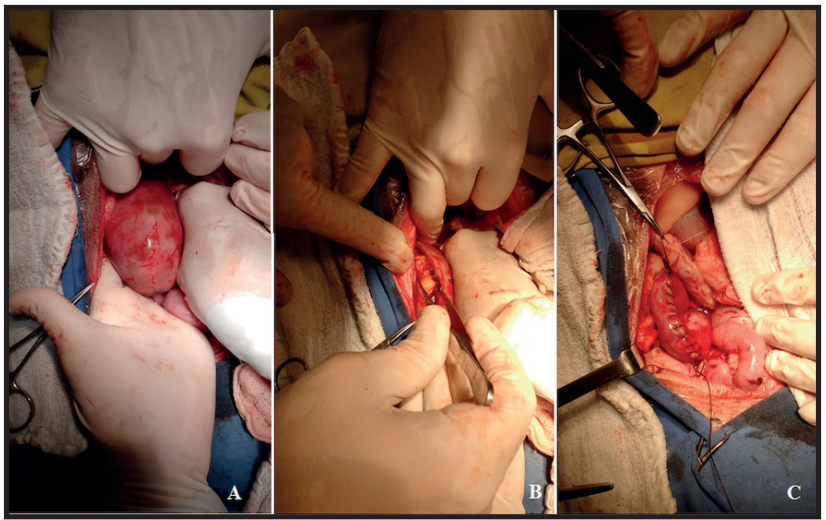

Figure 2. A- Markedly enlarged and firm kidney during surgery. B- Urolith view after kidney incision. C- Incision closure with a continuous pattern of absorbable suture material.

organ. Digital occlusion of the renal artery and vein was performed, and the organ was incised on its convex surface. The incision was extended by approximately eight centimeters and the urolith was gently removed (Figure 2B). Due to the fragility of renal tissue, the kidney incision was closed with a continuous pattern of absorbable suture material (Polyglactin 910 n. 2-0 and atraumatic needle $)^{7}$ [Figure $2 \mathrm{C}$ ]. The renal vessels were released and no bleeding was noticed on the suture line. The kidney was then omentalized and the abdominal cavity was closed routinely. The right nephrolith measured about $9 \times 7 \mathrm{~cm}$ (Figure 1C). The sample was sent for quantitative analysis to the Minnesota Urolith Center (St. Paul, Minnesota, USA) and the stone was composed of $100 \%$ struvite and contained no nidus.

After surgery, the dog remained hospitalized during five days and presented a stable clinical condition. Blood creatinine in the postoperative period was $274 \mu \mathrm{mol} / \mathrm{L}$ and hematocrit was $16 \%$. Another whole blood transfusion was performed, and human recombinant erythropoietin ${ }^{8}$ therapy was started (100 UI/kg SC) every $48 \mathrm{~h}$. The patient was discharged and the same therapy was maintained, in addition to weekly clinical and laboratory follow-up. Despite clinical stability, the dog presented constant azotemia and hematuria, and new blood transfusions were necessary to maintain hematocrit. After three months, nephrotomy of the left kidney was performed to remove the other urolith, using the same surgical technique described. The left nephrolith measured about 9 × $8 \mathrm{~cm}$ (Figure 1D). Another postoperative whole blood transfusion was performed and the dog was hospitalized for supportive therapy. Antibiotic therapy with amoxicillin/potassium clavulanate $^{9}(25 \mathrm{mg} / \mathrm{kg} \mathrm{q} 12 \mathrm{~h} \mathrm{PO})$ and metronidazole ${ }^{10}$

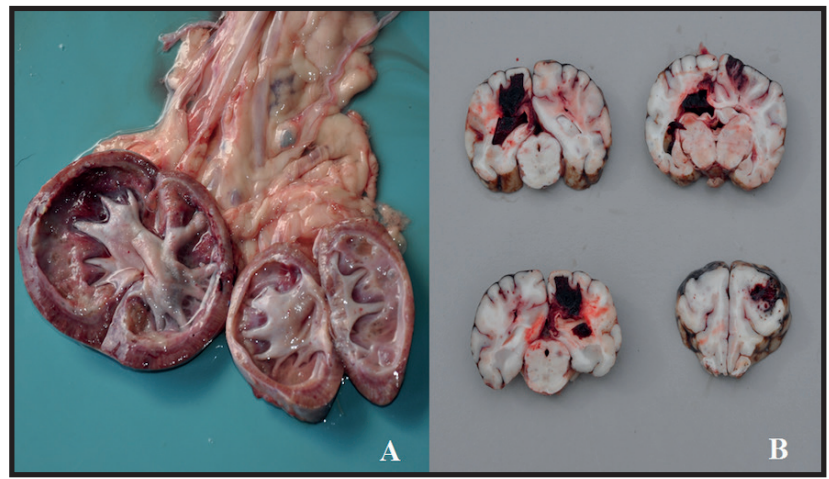

Figure 3. Necropsy examination. A- Extensive loss of renal parenchyma in both kidneys. B- Presence of thrombus and hemorrhagic areas in the central nervous system.

(20 mg/kg q 12h PO) was instituted. The patient was clinically stable and with no macroscopically hematuria $12 \mathrm{~h}$ after surgery. In five days the dog was discharged, maintaining the same therapy.

Two days after discharge, the patient returned prostrated in lateral recumbency, however with no alteration of parameters on physical examination. Venous blood gas, $\mathrm{pH}$ and electrolyte analysis showed no major alteration, besides mild metabolic acidosis. Blood tests showed anemia (hematocrit 18\%), thrombocytopenia $(10,000$ platelets $\mu \mathrm{L})$, hypoalbuminemia (16 g/L), azotemia (creatinine $335.9 \mu \mathrm{mol} / \mathrm{L}$ and urea $31.3 \mathrm{mmol} / \mathrm{L})$ and hyperphosphatemia $(2.3 \mathrm{mmol} / \mathrm{L})$. Fresh frozen plasma was transfused for correction of hypoalbuminemia. The dog presented a convulsion and died shortly, eight hours later. In the necropsy examination, extensive loss of renal parenchyma was seen in both kidneys (Figure 3A) and the presence of thrombus and areas of infarction in several organs such as spleen, liver, lungs and central nervous system (Figure 3B).

\section{DISCUSSION}

Nephrolithiasis may be associated with recurrent urinary tract infections in dogs [3]. The presence of bacteria that produce urease, eg. Staphylococcus spp., increases urine $\mathrm{pH}$, which may precipitate struvite crystals and the formation of this type of urolith [6], as in this case. The diagnosis of chronic kidney disease obtained by the clinical, imaging and laboratory findings in this case explains the presence of non-regenerative anemia, since anemia of chronic kidney disease occurs due to decreased production of erythropoietin by the kidneys [8]. In the present case, serial blood transfusions were required to correct 
anemia, caused by persistent hematuria in association with renal disease.

Only those nephroliths contributing to outflow obstruction, recurrent infection, pain, and those enlarging to the point of causing renal parenchymal compression, should be considered for removal [4]. Surgical removal is also indicated in cases in which the time required for clinical dissolution of the urolith causes more renal lesion than the surgical procedure [10]. According to the ACVIM Small Animal Consensus Recommendations on the Treatment and Prevention of Uroliths in Dogs and Cats [4], problematic nephroliths should be removed by minimally invasive procedures. Extracorporeal shock wave lithotripsy, endoscopic nephrolithotomy or clinical dissolution of struvite uroliths are the most recommended techniques [4]. However, in this case, such procedures were not indicated because of the size of the urolith. In addition, the clinical condition of the patient required faster treatment, therefore, nephrotomy was chosen.

Nephrotomy usually is performed to remove uroliths in the renal pelvis, but also to explore the renal pelvis for neoplasia or persistent hematuria [5]. Removal of the nephrolith may decrease the progression of renal injury [11]. Nephrotomy may be done by bisecting the kidney or by using an intersegmental approach, in which the interlobar arteries are not transected, minimizing nephron destruction [5]. A study performed in normal dogs comparing both techniques concluded that neither one affected glomerular filtration rate, but because bisection approach requires less surgical manipulation and time, it is preferred [12]. For this reason, in this case, the bisection approach was chosen. Occlusion of renal vessels was performed with assistant's fingers. The aim is to interrupt the blood flow of the organ, helping to contain the trans-surgical hemorrhage. Renal ischemic time should be less than 20 minutes, otherwise renal injury may be caused [3]. A study in swines showed that clamping renal artery alone results in less ischemic damage of the organ [2], however, dissection to separate the renal artery and vein is necessary [3], increasing surgical time.

The decision to perform unilateral or bilateral nephrotomy depends on the patient's renal function and general clinical condition, degree of obstruction and the presence of pyelonephritis [13]. Bilateral approach may promote acute kidney injury if renal function is sufficiently compromised preoperatively [5]. Staged procedures can be performed four to six weeks apart [3]. In these cases, it is recommended to operate the less affected kidney first, since it is less likely to occur anesthetic and surgical complications [13]. For this patient, the nephrotomies were performed three months apart, due to the diagnosis of chronic kidney disease and the persistent azotemia.

Complications of nephrotomy include perirenal hemorrhage, hydronephrosis due to obstruction of blood clots [13] or urine leakage to the abdominal cavity [3]. From the necropsy findings, the surgical site did not present these complications, only omental adhesions were observed, justifying that the surgical procedure was not directly related to death. In addition, the patient remained clinically stable in the immediate postoperative of both procedures and was discharged on both occasions. Correction of dehydration, blood transfusion, and antibiotic therapy based on urine culture and susceptibility testing was important for initial stabilization, before surgery. However, the presence of advanced chronic kidney disease may have contributed to deterioration of the patient's clinical condition and death. Even with the maintenance of antimicrobial therapy, the patient had constant signs of urinary infection, such as leukocytosis and bacteriuria/pyuria. The presence of uroliths is associated with complicated bacterial infection, due to the incorporation of the bacteria in its matrix, which prevents the penetration and proper action of the antimicrobials [6].

The presence of thrombus and hemorrhagic areas, observed during necropsy, associated with acute onset of clinical signs and the presence of severe hypoalbuminemia, may suggest that the patient died due to a thromboembolic event. Nephrotic syndrome is an uncommon complication of protein-losing nephropathies in dogs. It refers to the presence of proteinuria, hypoalbuminemia, hyperlipidemia and extravascular accumulation of fluids. All patients with proteinuria are at increased risk of thromboembolic complications due to urinary loss of anticoagulant proteins, increased synthesis of procoagulant factors and generalized platelet activation [9].

Nephrotomy can be performed with few adverse effects on renal function if the surgical technique and anesthetic management are adequate. Removal of nephroliths may decrease the progression of renal injury, and be beneficial in controlling pain, hematuria, and associated urinary tract infection. In this case, 
nephrotomy was the best option for the treatment of the patient, since it enabled a rapid intervention, controlled the hematuria and allowed the removal of both uroliths without complications related to surgery.

\section{MANUFACTURERS}

${ }^{1}$ JP Indústria Farmacêutica SA. Ribeirão Preto, SP, Brazil.

${ }^{2}$ Medley Farmacêutica Ltda. São Paulo, SP, Brazil.

${ }^{3}$ Zoetis Indústria de Produtos Veterinários Ltda. Campinas, SP, Brazil.

${ }^{4}$ Laboratórios Pfizer Ltda. Itapevi, SP, Brazil.
${ }^{5}$ Ouro Fino Saúde Animal SA. Cravinhos, SP, Brazil.

${ }^{6}$ Cristália Produtos Químicos Farmacêuticos Ltda. São Paulo, SP, Brazil.

${ }^{7}$ Johnson \& Johnson do Brasil Indústria e Comércio Ltda. São Paulo, SP, Brazil.

${ }^{8}$ Aché Laboratórios Farmacêuticos SA. São Paulo, SP, Brazil. ${ }^{9}$ União Química Farmacêutica Nacional SA. São Paulo, SP, Brazil.

${ }^{10}$ Laboratório Teuto Brasileiro SA. Anapolis, GO, Brazil.

Declaration of interest. The authors declare no conflicts of interest. The authors alone are responsible for the content and writing of this paper.

\section{REFERENCES}

1 Bartges J.W. \& Callens A.J. 2015. Urolithiasis. Veterinary Clinics of North America: Small Animal Practice. 45(4): 747-768. DOI: 10.1016/j.cvsm.2015.03.001.

2 Colli J.L., Wang Z., Johnsen N., Grossman L. \& Lee B.R. 2013. Clamping renal artery alone produces less ischemic damage compared to clamping renal artery and vein together in two animal models: near-infrared tissue oximetry and quantitation of 8-isoprostane levels. International Urology Nephrology. 45(2): 421-428. DOI: 10.1007/s11255-0120297-7.

3 Lanz O.I. \& Waldron D.R. 2000. Renal and Ureteral Surgery in Dogs. Clinical Techniques in Small Animal Practice. 15(1): 1-10. DOI: $10.1053 / \mathrm{svms} .2000 .7299$.

4 Lulich J.P., Berent A.C., Adams L.G., Westropp J.L., Bartges J.W. \& Osborne C.A. 2016. ACVIM Small Animal Consensus Recommendations on the Treatment and Prevention of Uroliths in Dogs and Cats. Journal of Veterinary Internal Medicine. 30(5): 1564-1574. DOI: 10.1111/jvim.14559.

5 MacPhail C.M. 2013. Surgery of the Kidney and Ureter. In: Fossum T.W., Dewey C.W., Horn C.V., Johnson A.I., MacPhail C.M., Radlinsky M.G., Schulz K.S. \& Willard M.D. (Eds). Small Animal Surgery. Chapter 25. 4th edn. St Louis: Elsevier, pp.705-734.

6 Olin S.J. \& Bartges J.W. 2015. Urinary Tract Infections Treatment/Comparative Therapeutics. Veterinary Clinics of North America: Small Animal Practice. 45(4): 721-746. DOI: 10.1016/j.cvsm.2015.02.005.

7 Osborne C.A., Lulich J.P., Kruger J.M., Ulrich L.K. \& Koehler L.A. 2009. Analysis of 541,891 Canine Uroliths, Feline Uroliths, and Feline Urethral Plugs from Perspectives from the Minnesota Urolith Center. Veterinary Clinics of North America: Small Animal Practice. 39(1): 183-197. DOI: 10.1016/j.cvsm.2008.09.011.

8 Polzin D.J. 2011. Chronic Kidney Disease in Small Animals. Veterinary Clinics of North America: Small Animal Practice. 41(1): 15-30. DOI: 10.1016/j.cvsm.2010.09.004.

9 Pressler B. 2011. Nephrotic syndrome. In: Bartges J. \& Polzin D.J. (Eds). Nephrology and Urology of Small Animals. Chapter 44. West Sussex: Wiley-Blackwell, pp.415-421.

10 Ross S.J., Osborne C.A., Lulich J.P., Polzin D.J., Ulrich L.K., Koehler L.A., Bird K.A. \& Swanson L.L. 1999. Canine and Feline Nephrolithiasis: Epidemiology, Detection, and Management. Veterinary Clinics of North America: Small Animal Practice. 29(1): 231-250. DOI: 10.1016/s0195-5616(99)50013-2.

11 Stone E.A. \& Gookin J.D. 2000. Indications for nephrectomy and nephrotomy. In: Bonagura J.D. (Ed). Kirk's Current Veterinary Therapy XIII. 13th edn. Philadelphia: Saunders, pp.866-868.

12 Stone E.A., Robertson J.L. \& Metcalf M.R. 2002. The Effect of Nephrotomy on Renal Function and Morphology in Dogs. Veterinary Surgery. 31(4): 391-397. DOI: 10.1053/jvet.2002.33.595.

13 Tobias K. 2011. Renal and ureteral surgery. In: Bartges J. \& Polzin D.J. (Eds). Nephrology and Urology of Small Animals. Chapter 60. West Sussex: Wiley-Blackwell, pp.596-616. 